\title{
Nonlinear Identification and Control of Coupled Mass-Spring-Damper System using Polynomial Structures
}

\author{
Sana RANNEN \\ Laboratory of Advanced Systems \\ Polytechnic High School of Tunisia \\ LSA - EPT \\ University of Carthage \\ BP 743, 2078 La Marsa
}

\author{
Chekib GHORBEL \\ Laboratory of Advanced Systems \\ Polytechnic High School of Tunisia \\ LSA - EPT \\ University of Carthage \\ BP 743, 2078 La Marsa
}

\author{
Naceur BENHADJ BRAIEK \\ Laboratory of Advanced Systems \\ Polytechnic High School of Tunisia \\ LSA - EPT \\ University of Carthage \\ BP 743, 2078 La Marsa
}

\begin{abstract}
The paper aims to identify and control the coupled mass-spring-damper system. A nonlinear discrete polynomial structure is elaborated. Its parameters are estimated using Recursive Least Squares (RLS) algorithm. Moreover, a feedback stabilizing control law based on Kronecker power is designed. Finally, simulations are presented to illustrate the effectiveness of the proposed structure.
\end{abstract}

Keywords-Identification; RLS algorithm; Polynomial structure; Stabilizing control; $L Q R$

\section{INTRODUCTION}

System identification is an important tool which can be used to improve control performance [1] [2]. It is the process of developing a mathematical representation of a physical system based on observed data with sufficient accuracy.

Identification of complex systems has stilled a major problem in automatic control because there is no general method for studying high order processes. Indeed, it has received considerable attention and several types of models have been proposed during the last decades [3] [4] [5] [6] [7]. Such as Volterra model [8] [9], Wiener model [10], Hammerstein model [11], Nonlinear AutoregRessive with eXogenous input (NARX) model [12], Nonlinear AutoregRessive Moving Average with eXogenous input (NARMAX) model [13] [14], etc. However the elaboration of a suitable feedback stabilizing control using the proposed models remain difficult.

Nonlinear discrete polynomial structure is general enough to describe many physical systems [15] [16]. It presents the advantage to permit the use of the kronecker product and power of matrices and vectors, which allows important algebraic manipulations [17]. Moreover, it allowed to design a feedback stabilizing control law [18].

In this work, a suitable nonlinear discrete polynomial structure was elaborated. Recursive Least Square (RLS) algorithm is used for parameters estimation. The polynomial model allowed to design an efficient feedback stabilizing control law. A CMSD system illustrated the proposed nonlinear parametric estimation and structures.

This paper is organized as First, the nonlinear identification procedure is defined. Second, the feedback stabilizing control is presented. Third, the proposed identification method is applied to CMSD system and finally a conclusion is made.

\section{System IDENTIFICATION}

In automatic control applications, a compact and accurate description of the dynamic behavior of the system under consideration is needed. Nonlinear models can be constructed from theoretical modeling on the basis of a priori knowledge on the nature of the systems. However, these white-box models are very complex and difficult to derive because they require detailed specialist knowledge which is practically or totally unavailable in practical situation [19].

An alternative way of building models is by system identification. It is the process of improving a mathematical representation of a physical systems based on observed input/output data with sufficient accuracy which can be used to improve control performance and achieve robust fault tolerant behavior.

The identification procedure is summarized as follows:

- collection of the inputs and outputs measurements,

- selection of the model,

- choice of the identification algorithm in order to estimate the parameters that describe the model,

- validity of the obtained model is evaluated.

There are several types of models that describe complex systems. Nonlinear discrete polynomial structures is one of the most performers models. Hence, it can approach with satisfactory accuracy any analytical nonlinear system and thus ensure the mathematical description of a wide range of physical process [18] [20] [15]. Moreover, the description of polynomial systems can be simplified using the Kronecker product and power vectors and matrices.

\section{A. Nonlinear discrete polynomial structures}

We consider in this paper the discrete nonlinear polynomial systems described by a state equation of the following form [16]:

$$
X_{k+1}=F\left(X_{k}\right)+G\left(X_{k}\right) U_{k}
$$


where $F\left(X_{k}\right)$ and $G\left(X_{k}\right)$ are a polynomials vectors functions. They are given by [15]:

$$
\begin{gathered}
F\left(X_{k}\right)=\sum_{i \geq 1} A_{i} X_{k}^{[i]} \\
G\left(X_{k}\right)=\sum_{i \geq 0} B_{i}\left(I_{m} \otimes X_{k}^{[i]}\right)
\end{gathered}
$$

with $X_{k}=\left(x_{1, k}, x_{2, k}, \ldots, x_{n, k}\right)^{T} \in R^{n}, X_{k}^{[i]}$ is the Kronecker power of the vector $X_{k}$ defined as:

$$
\left\{\begin{array}{l}
X_{k}^{[0]}=1 \\
X_{k}^{[i]}=X_{k}^{[i-1]} \otimes X_{k}=X_{k} \otimes X_{k}^{[i-1]} \\
\text { for } i \geq 1
\end{array}\right.
$$

where $\otimes$ designates the symbol of the Kronecker product, $A_{i}$ and $B_{i}$ are respectively $\left(n \times n^{i}\right)$ and $\left(n \times m n^{i}\right)$ matrices. $I_{m}$ is the identity matrix of order $m$. We assume that the pair $\left(A_{1}, B_{0}\right)$ is completely controllable.

Parametric estimation using recursive algorithms is one of the most important areas in system and signal processing. The RLS algorithm is one of the most popular ones and widely used for the parameter estimation because of his capability to approximate a large class of systems and his simplicity of implementation [21].

\section{B. RLS algorithm}

RLS algorithm allows to estimate the model parameters by minimizing a measure of the model prediction error given by [22]:

$$
\varepsilon_{k}=y_{k}-\hat{y}_{k}
$$

where $\hat{y}_{k}$ is the prediction of the scalar measured output $y_{k}$. It is given by:

$$
\hat{y}_{k}=\hat{\theta}_{k}^{T} \psi_{k}
$$

$\hat{\theta}_{k}$ is the vector of estimated parameters and $\psi_{k}$ is the regression vector containing old inputs and outputs of the system to be identified.

The RLS algorithm can be written in following form:

$$
\left\{\begin{array}{l}
\hat{\theta}_{k}=\hat{\theta}_{k-1}+P_{k} \psi_{k} \varepsilon_{k} \\
P_{k}=P_{k-1}-\frac{P_{k-1} \psi_{k} \psi_{k}^{T} P_{k-1}}{1+\psi_{k}^{T} P_{k} \psi_{k}} \\
\varepsilon_{k}=y_{k}-\hat{y}_{k}
\end{array}\right.
$$

with $P_{k}$ is the gain matrix. It is given by:

$$
P_{k}=\left(\sum_{i=n+1}^{k} \psi_{i} \psi_{i}^{T}\right)^{-1}
$$

\section{Performance indicators}

The performance of the models is assessed using the Mean Square Error (MSE) and the Variance-Accounted-For (VAF) indicators [12]:

$$
\begin{gathered}
M S E=\frac{1}{N} \sum_{k=1}^{N}\left(y_{s, k}-y_{k}\right)^{2} \\
V A F=\max \left\{1-\frac{\operatorname{var}\left(y_{s, k}-y_{k}\right)}{\operatorname{var}\left(y_{s, k}\right)}, 0\right\} \times 100
\end{gathered}
$$

where $y_{s, k}$ and $y_{k}$ are respectively the system and the model output, $N$ present the number of iterations and $\operatorname{var}($. denotes the variance of a signal.

\section{Nonlinear Feedback Stabilizing Control}

In this section, we propose to determine a stabilizing control law of the system in the following form [18]:

$$
U_{k}=H\left(X_{k}\right)
$$

where $H\left(X_{k}\right)$ is an analytical vectorial function from $R^{n}$ into $R^{m}$

It is expressed by generalized Taylor series:

$$
H\left(X_{k}\right)=-\sum_{j \geq 1} K_{j} X_{k}^{[i]}
$$

where $K_{j}, j=1, \ldots, r$ are $\left(m \times n^{i}\right)$ matrices. Thus, the controlled system equation can be written as [18]:

$$
\begin{aligned}
X_{k+1} & =\sum_{i \geq 1} A_{i} X_{k}^{[i]} \\
& -\sum_{i \geq 0} \sum_{j \geq 0} B_{i}\left(I_{m} \otimes X_{k}^{[i]}\right) K_{j} X_{k}^{[j]}
\end{aligned}
$$

Our objective is to determine the control function so that the stability of the null equilibrium $\left(X_{k}=0\right)$ of the system. The best solution of such a problem consists in the determination of the matrices $K_{j}, j \in N$. The matrix $K_{1}$ is obtained using the Discrete Linear Quadratic Regulator (DLQR) state feedback design.

DLQR is one of the optimal control techniques. It takes into account the states of the dynamical system and control input to make the optimal control decisions. This is simple as well as robust [23] [24]. The discrete state equation is given by:

$$
X_{k+1}=A_{1} X_{k}+B_{0} U_{k}
$$

then, the state feedback control $U_{k}$ is defined as:

$$
U_{k}=-K_{1} X_{k}
$$

which leads to:

$$
X_{k+1}=\left(A_{1}-B_{0} K_{1}\right) X_{k}
$$

$K_{1}$ is derived from minimization of the cost function:

$$
J\left(X_{k}\right)=\frac{1}{2} \sum_{i=k}^{\infty}\left(X_{i}^{T} Q X_{i}+U_{i}^{T} R U_{i}\right)
$$


where $Q$ and $R$ are positive semi-definite and positive definite symmetric constant matrices, respectively. The DLQR gain vector $K_{1}$ is given by:

$$
K_{1}=\left(R+B_{0}^{T} P B_{0}\right)^{-1} B_{0}^{T} P A_{1}
$$

where $P$ is a positive definite symmetric constant matrix obtained from the solution of matrix Algebraic Riccati Equation (ARE):

$$
\begin{gathered}
A_{1}^{T} P A_{1}-P+Q \\
-A_{1}^{T} P B_{0}\left(R+B_{0}^{T} P B_{0}\right)^{-1} B_{0}^{T} P A_{1}=0
\end{gathered}
$$

However, the matrices $K_{j}$, for $j \geq 2$, are given by the following relation [18]:

$$
K_{j}=-B_{0}^{+}\left(A_{j}+\sum_{i=1}^{j-1} B_{i}\left(K_{1-i} \otimes I_{n^{i}}\right)\right)
$$

where $B_{0}^{+}$designates the Moore-Penrose pseudo-inverse of the matrix $B_{0}$.

\section{Illustrative EXAMPLE: COUPLED MASS-SPRING-DAMPER SYSTEM}

\section{A. CMSD system description}

The CMSD system, shown in Figure 1, is composed of two nonlinear springs, two weights and two dampers. Since the upper mass $m_{1}$ is attached to both springs, there are two nonlinear springs restoring forces acting upon it: an upward force $f_{r 1}$ exerted by the elongation, or compression, $x_{1}$ of the first spring; an upward force $f_{r 2}$ from the second spring resistance to being elongated, or compressed, by the amount $\left(x_{2}-x_{1}\right)$.

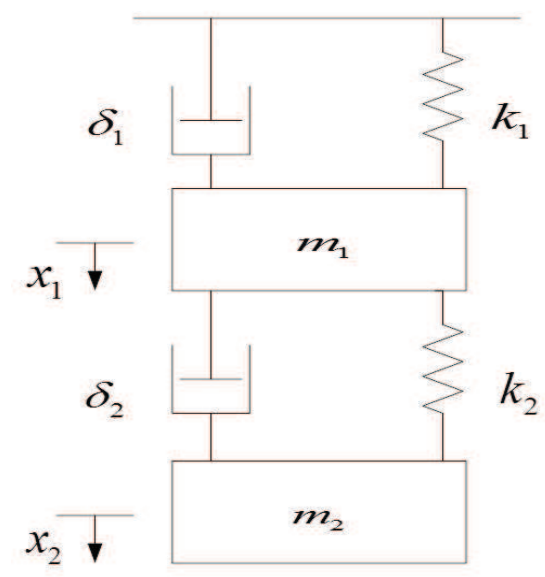

Fig. 1 - Mechanical model of the CMSD system

The second mass $m_{2}$ only feels the nonlinear restoring force from the elongation, or compression, of the second spring. Allowing the system to come and to rest in equilibrium, we measure the displacement of the center of mass of each weight from equilibrium, as a function of time, and denote these measurement by $x_{1}$ and $x_{2}$ respectively. System parameters are presented in Table 1 [25].
TABLE I - Parameter Description of CMSD System

\begin{tabular}{l||l|l}
\hline Parameter & Description & Value \\
\hline$k(N / m)$ & spring constant & $k_{1}=\frac{2}{5}, k_{2}=1$ \\
$x(m)$ & displacement & $x_{1}, x_{2}$ \\
$m(K g)$ & mass of the weight & $m_{1}=1, m_{2}=2$ \\
$\delta(N s / m)$ & damping coefficient & $\delta_{1}=\frac{1}{10}, \delta_{2}=\frac{1}{5}$ \\
$\mu$ & nonlinear coefficient & $\mu_{1}=\frac{1}{6}, \mu_{2}=\frac{1}{10}$ \\
\hline
\end{tabular}

1) Mathematical model: The continuous nonlinear equations of the CMSD system are given by:

$$
\left\{\begin{array}{c}
m_{1} \ddot{x}_{1}=-\delta_{1} \dot{x}_{1}-k_{1} x_{1}+\mu_{1} x_{1}^{3}-k_{2}\left(x_{1}-x_{2}\right) \\
+\mu_{2}\left(x_{1}-x_{2}\right)^{3}+u_{1} \\
m_{2} \ddot{x}_{2}=-\delta_{2} \dot{x}_{2}-k_{2}\left(x_{2}-x_{1}\right) \\
+\mu_{2}\left(x_{2}-x_{1}\right)^{3}+u_{2}
\end{array}\right.
$$

2) Proposed identification and feedback stabilizing control using polynomial structures: The proposed nonlinear discrete polynomial structure that describes perfectly our system is as follow, the sampling time $T_{e}=0.01 \mathrm{~s}$ and the initial conditions of the state variables

$X_{k}(0)=\left(\begin{array}{cccc}0.7 & 0 & 0.1 & 0\end{array}\right)^{T}$, with $x_{1, k}$ displacement of the first mass, $\Omega_{1, k}$ velocity of the first mass, $x_{2, k}$ displacement of the second mass and $\Omega_{2, k}$ velocity of the second mass:

$$
X_{k+1}=A_{1} X_{k}+A_{2} X_{k}^{[2]}+\left(B_{0}+B_{1} X_{k}\right) U_{k}
$$

with:

$$
\begin{aligned}
& X_{k}=\left(\begin{array}{c}
x_{1, k} \\
\Omega_{1, k} \\
x_{2, k} \\
\Omega_{2, k}
\end{array}\right), U_{k}=\left(\begin{array}{c}
u_{1, k} \\
u_{2, k}
\end{array}\right) \\
& A_{1}=\left(\begin{array}{cccc}
a_{11} & a_{12} & a_{13} & a_{14} \\
a_{21} & a_{22} & a_{23} & a_{24} \\
a_{31} & a_{32} & a_{33} & a_{34} \\
a_{41} & a_{42} & a_{43} & a_{44}
\end{array}\right),
\end{aligned}
$$

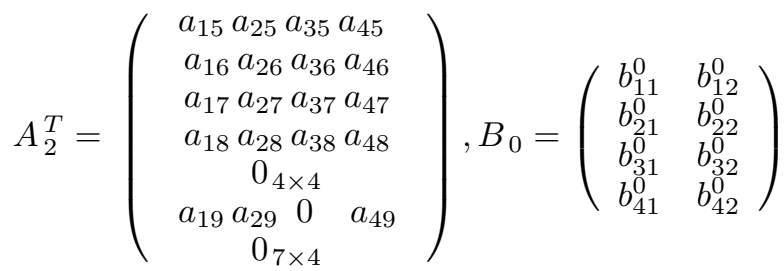$$
\text { and } B_{1}=\left(\begin{array}{cccccccc}
b_{11}^{1} & b_{12}^{1} & b_{13}^{1} & b_{14}^{1} & b_{11}^{2} & b_{12}^{2} & b_{13}^{2} & b_{14}^{2} \\
b_{21}^{1} & b_{22}^{1} & b_{23}^{1} & b_{24}^{1} & b_{21}^{2} & b_{22}^{2} & b_{23}^{2} & b_{24}^{2} \\
b_{31}^{1} & b_{32}^{1} & b_{33}^{1} & b_{34}^{1} & b_{31}^{2} & b_{32}^{2} & b_{33}^{2} & b_{34}^{2} \\
b_{41}^{1} & b_{42}^{1} & b_{43}^{1} & b_{44}^{1} & b_{41}^{2} & b_{42}^{2} & b_{43}^{2} & b_{44}^{2}
\end{array}\right) \text {. }
$$

The performance of the proposed polynomial structure is assessed using the MSE and the VAF indicators, is presented in Table 2. 
TABLE II - Performance Indicators

\begin{tabular}{l||l|l}
\hline & $M S E$ & VAF \% \\
\hline$x_{1, k}$ & $1.583310^{-9}$ & 99.8068 \\
$\Omega_{1, k}$ & $5.926910^{-13}$ & 99.9999 \\
$x_{2, k}$ & $1.194910^{-9}$ & 93.7454 \\
$\Omega_{2, k}$ & $1.737410^{-10}$ & 99.9608 \\
\hline
\end{tabular}

To stabilize the CMSD system, we consider the following nonlinear control law:

$$
U_{k}=-K_{1} X_{k}-K_{2} X_{k}^{[2]}
$$
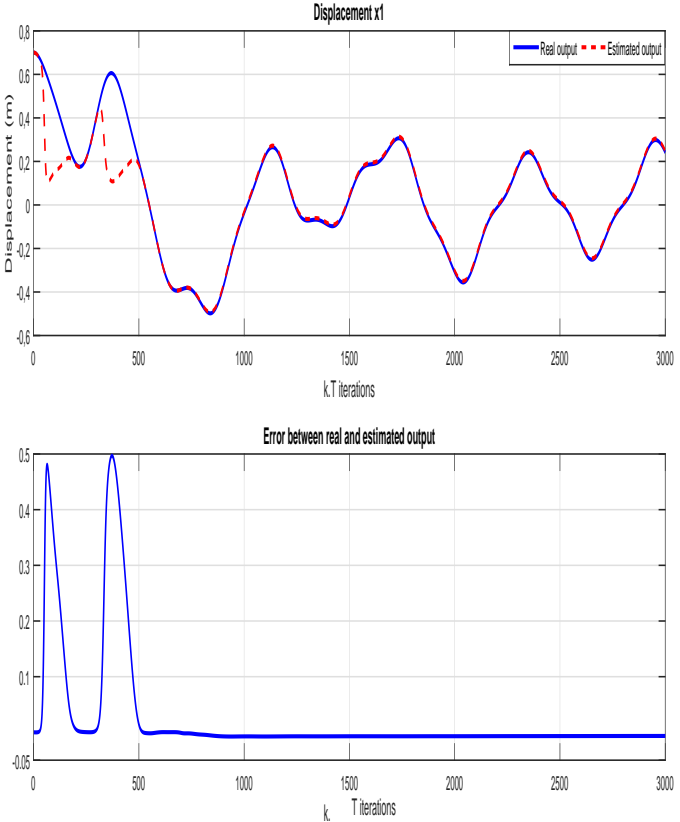

Fig. 2 - Displacement of the first mass $x_{1, k}$ in the open-loop

with:

$$
K_{1}=\left(\begin{array}{llll}
8.2253 & 10.2009 & 0.7640 & -0.0844 \\
0.9997 & 0.1870 & 8.1414 & 9.2730
\end{array}\right)
$$

$$
\text { and } K_{2}^{T}=\left(\begin{array}{cc}
-0.2407 & 0.0139 \\
-0.1001 & 0.0322 \\
0.1504 & -0.0529 \\
0.0119 & -0.0044 \\
-0.1708 & -0.0004 \\
-0.0791 & 0.0034 \\
0.0645 & 0 \\
0 & 0 \\
0.0003 & 0.0106 \\
-0.0059 & 0.1479 \\
0.0048 & 0.0004 \\
0 & 0 \\
0.0014 & -0.0190 \\
0.0007 & 0.1685 \\
-0.0005 & 0.0005 \\
0 & 0
\end{array}\right) \text {. }
$$

3) Simulation results: For parameters estimation of CMSD system, we choose the causal signals $u_{1, k}=\frac{1}{3} \sin \left(k \pi T_{e}\right)$ and $u_{2, k}=\frac{1}{5} \sin \left(k \pi T_{e}\right)$, as inputs of the CMSD system.

The responses of real and estimated state variables $x_{1, k}$ and $x_{2, k}$, as well as, the errors are presented from Figures 2 and 3 , respectively.
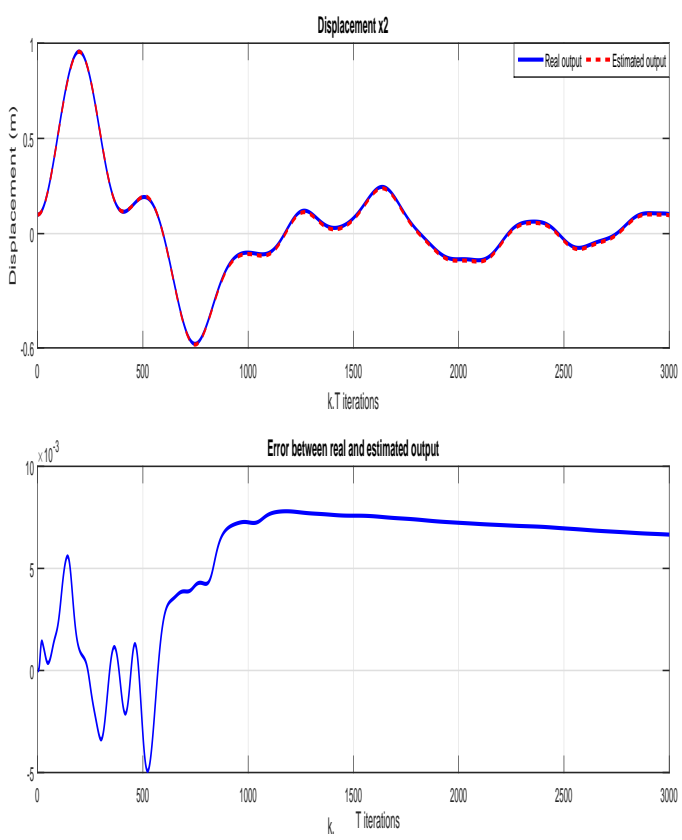

Fig. 3 - Displacement of the second mass $x_{2, k}$ in the open-loop

Figure 4 shows the control signals $u_{1, k}$ and $u_{2, k}$. The responses of the state variables $x_{1, k}$ and $x_{2, k}$ of the CMSD system using nonlinear feedback stabilizing control technique, equation 23, are depicted in Figure 5. 

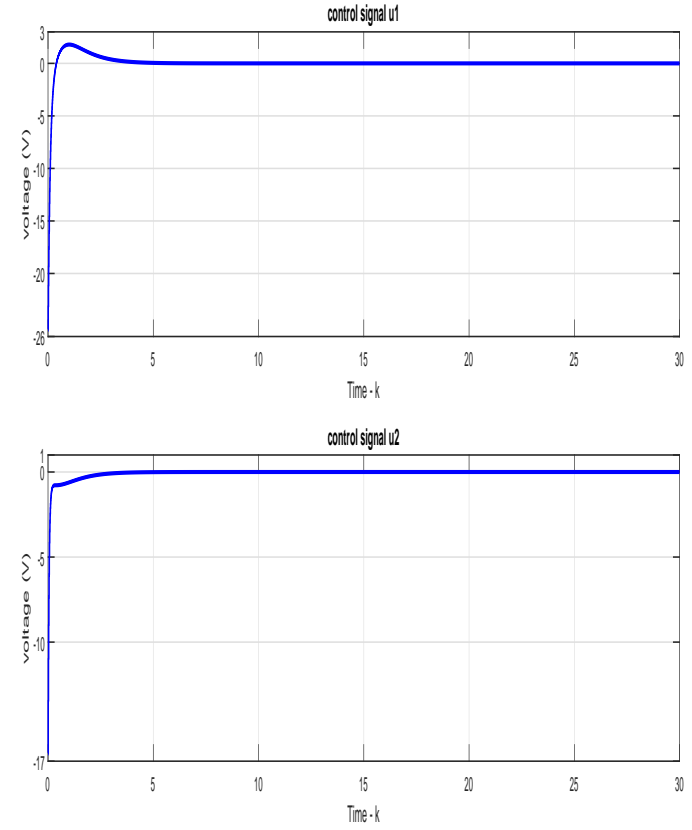

Fig. 4 - Control signals $u_{1, k}$ and $u_{2, k}$
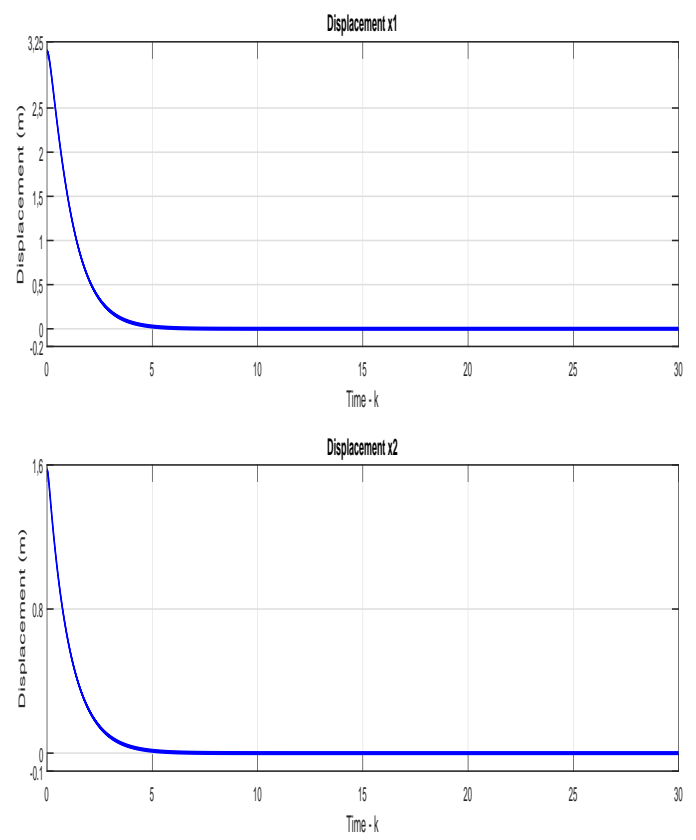

Fig. 5 - Closed-loop displacements $x_{1, k}$ and $x_{2, k}$ evolution

\section{DISCUSSION}

The main concern of the paper was to determine suitable nonlinear discrete polynomial structure of complex systems, which allowed to design a feedback stabilizing control law.

As can be seen from Figures 2 and 3, the identified outputs tracks the behavior of the real ones perfectly. The modeling errors range of $x_{1, k}$ and $x_{2, k}$ are from -0.05 to 0.5 and -0.001 to 0.005 , respectively. As well as, the indicator performance values given in Table 2 , the elaborate model applied to the CMSD system can achieve a sufficiently high modeling accuracy.

The convergence of the nonlinear discrete polynomial model parameters values obtained using the RLS algorithm is presented, in Appendix A, Table 3. Indeed, Figure 4 shows that by applying the proposed structure to design feedback gains based on Kronecker power, suitable inputs can be produced for CMSD system that make state variables track equilibrium point rapidly, as given in Figure 5.

\section{CONCLUSION}

A nonlinear discrete polynomial structure has been elaborated. RLS algorithm has been used for the parameters estimation. The polynomial structure allowed to design a feedback stabilizing control law based on Kronecker power for complex systems. The proposed structure has been applied successfully to model and stabilize CMSD system.

Simulation results demonstrate that the identified model has allowed to elaborate a feedback stabilizing control law, which had provided a satisfactory performance in stabilizing the CMSD system at the equilibrium points.

\section{APPENDIX A}

TABLE III - Polynomial Structure Parameters Values

\begin{tabular}{llll}
\hline Iterations & $k=1000$ & $k=2500$ & $k=3000$ \\
\hline$a_{11, k}$ & 0.8964 & 0.9889 & 0.9999 \\
$a_{12, k}$ & 0.0064 & 0.0094 & 0.0100 \\
$a_{13, k}$ & $5.071110^{-} 5$ & $5.064710^{-} 5$ & $5.066810^{-} 5$ \\
$a_{14, k}$ & $2.437510^{-} 7$ & $3.693110^{-} 7$ & $5.157810^{-} 7$ \\
$a_{15, k}$ & $6.941910^{-} 6$ & $6.937110^{-} 6$ & $7.024710^{-} 6$ \\
$a_{16, k}$ & $3.720710^{-} 7$ & $6.914910^{-} 7$ & $1.061610^{-} 6$ \\
$a_{17, k}$ & $-3.894310^{-} 6$ & $-3.917810^{-} 6$ & $-4.110610^{-} 6$ \\
$a_{18, k}$ & $-4.473210^{-} 7$ & $-4.642010^{-} 7$ & $-4.924410^{-} 7$ \\
$a_{19, k}$ & $-2.012710^{-} 6$ & $-1.951010^{-} 6$ & $-1.883010^{-} 6$ \\
$a_{21, k}$ & -0.0116 & -0.0128 & -0.0136 \\
$a_{22, k}$ & 0.9858 & 0.9978 & 0.9988 \\
$a_{23, k}$ & 0.0088 & 0.0096 & 0.0099 \\
$a_{24, k}$ & $5.370410^{-} 5$ & $9.234110^{-} 5$ & $1.363210^{-} 4$ \\
$a_{25, k}$ & $9.931710^{-} 4$ & 0.0010 & 0.0010 \\
$a_{26, k}$ & $1.351010^{-} 4$ & $2.431710^{-} 4$ & $3.630010^{-} 4$ \\
$a_{27, k}$ & $-9.251210^{-} 4$ & $-9.390610^{-} 4$ & $-9.815810^{-} 4$ \\
$a_{28, k}$ & $-1.091110^{-} 4$ & $-1.124710^{-} 4$ & $-1.188910^{-} 4$ \\
$a_{29, k}$ & $-1.526510^{-} 4$ & $-1.447710^{-} 4$ & $-1.333710^{-} 4$ \\
$a_{31, k}$ & $9.953410^{-} 5$ & $9.953310^{-} 5$ & $9.953510^{-} 5$ \\
$a_{32, k}$ & $7.775810^{-} 8$ & $1.105010^{-} 7$ & $1.285910^{-} 7$ \\
$a_{33, k}$ & 0.9889 & 0.9987 & 0.9999 \\
$a_{34, k}$ & 0.005 & 0.008 & 0.01 \\
$a_{35, k}$ & $-4.845210^{-} 6$ & $-4.842310^{-} 6$ & $-4.827710^{-} 6$ \\
$a_{36, k}$ & $-3.535010^{-} 6$ & $-3.430510^{-} 6$ & $-3.295010^{-} 6$ \\
$a_{37, k}$ & $5.494410^{-} 6$ & $5.492210^{-} 6$ & $5.504610^{-} 6$ \\
$a_{38, k}$ & $-7.267710^{-} 7$ & $-6.197410^{-} 7$ & $-5.397510^{-} 7$ \\
$a_{41, k}$ & 0.0186 & 0.0190 & 0.0196 \\
$a_{42, k}$ & $5.710410^{-} 5$ & $5.660910^{-} 5$ & $5.562710^{-} 5$ \\
$a_{43, k}$ & -0.0184 & -0.0191 & -0.0194 \\
$a_{44, k}$ & 0.9948 & 0.9954 & 0.9958 \\
$a_{45, k}$ & $-3.096910^{-} 4$ & $-3.326910^{-} 4$ & $-3.326910^{-} 4$ \\
$a_{46, k}$ & $-2.255310^{-} 4$ & $-2.486110^{-} 4$ & $-2.834110^{-} 4$ \\
$a_{47, k}$ & 0.0008 & 0.0010 & 0.0011 \\
$a_{48, k}$ & $9.846510^{-} 4$ & $1.005910^{-} 5$ & $8.902010^{-} 5$ \\
$a_{49, k}$ & $-5.662710^{-} 4$ & $-5.589810^{-} 4$ & $-5.392810^{-} 4$ \\
\hline & & & \\
\hline
\end{tabular}




\begin{tabular}{|c|c|c|c|}
\hline Iterations & $k=1000$ & $k=2500$ & $k=3000$ \\
\hline$b_{11, k}^{0}$ & $4.971910^{-} 5$ & $4.975110^{-} 5$ & $4.979610^{-} 5$ \\
\hline$b_{12, k}^{0}$ & $-1.914510^{-} 7$ & $-1.729610^{-} 7$ & $-1.485910^{-} 7$ \\
\hline$b_{21, k}^{0}$ & 0.005 & 0.008 & 0.01 \\
\hline$b_{22, k}^{0}$ & $3.263810^{-} 4$ & $3.244010^{-} 4$ & $3.237610^{-} 4$ \\
\hline$b_{31, k}^{0}$ & $7.345010^{-} 8$ & $4.676210^{-} 8$ & $2.288010^{-} 8$ \\
\hline$b_{32, k}^{0}$ & $1.004910^{-} 4$ & $1.004610^{-} 4$ & $1.004210^{-} 4$ \\
\hline$b_{41, k}^{0}$ & $-2.296910^{-} 5$ & $-2.902310^{-} 5$ & $-3.513610^{-} 5$ \\
\hline$b_{42, k}^{0}$ & 0.0190 & 0.0194 & 0.0198 \\
\hline$b_{11, k}^{1}$ & $1.400110^{-} 6$ & $1.239210^{-} 6$ & $1.151910^{-} 6$ \\
\hline$b_{12, k}^{1}$ & $6.314510^{-} 6$ & $6.418810^{-} 6$ & $6.570010^{-} 6$ \\
\hline$b_{13, k}^{1}$ & $4.207710^{-} 7$ & $3.054110^{-} 7$ & $3.063010^{-} 7$ \\
\hline$b_{21, k}^{1}$ & $1.845110^{-} 4$ & $1.680210^{-} 4$ & $1.675110^{-} 4$ \\
\hline$b_{22, k}^{1}$ & $9.255810^{-} 5$ & $8.013910^{-} 5$ & $7.727810^{-} 5$ \\
\hline$b_{23, k}^{1}$ & $-8.019110^{-} 5$ & $-6.898110^{-} 5$ & $-6.333910^{-} 5$ \\
\hline$b_{31, k}^{1}$ & $10^{-} 6$ & $210^{-} 6$ & $210^{-} 6$ \\
\hline$b_{32, k}^{1}$ & $310^{-} 7$ & $410^{-} 7$ & $510^{-} 7$ \\
\hline$b_{41, k}^{1}$ & $3.810^{-} 4$ & $4.210^{-} 4$ & $4.410^{-} 4$ \\
\hline$b_{42, k}^{1}$ & $6.410^{-} 5$ & $7.210^{-} 5$ & $8.210^{-} 5$ \\
\hline$b_{11, k}^{2}$ & $-6.1710^{-} 6$ & $-5.6310^{-} 6$ & $-5.6410^{-} 6$ \\
\hline$b_{12, k}^{2}$ & $-6.1710^{-} 6$ & $-5.6310^{-} 6$ & $-5.4710^{-} 6$ \\
\hline$b_{13, k}^{2}$ & $5.0910^{-} 8$ & $1.4210^{-} 7$ & $1.4410^{-} 7$ \\
\hline$b_{21, k}^{2}$ & $-110^{-} 3$ & $-1.1810^{-} 3$ & $-1.310^{-} 3$ \\
\hline$b_{22, k}^{2}$ & $-210^{-} 4$ & $-310^{-} 4$ & $-410^{-} 4$ \\
\hline$b_{23, k}^{2}$ & $1.5410^{-} 5$ & $1.8610^{-} 5$ & $1.8210^{-} 5$ \\
\hline$b_{31, k}^{\overline{2}}$ & $3.8210^{-} 6$ & $4.310^{-} 6$ & $4.4510^{-} 6$ \\
\hline$b_{32, k}^{2}$ & $6.8610^{-} 7$ & $8.4610^{-} 7$ & $110^{-} 6$ \\
\hline$b_{41, k}^{2}$ & $610^{-} 4$ & $7.510^{-} 4$ & $910^{-} 4$ \\
\hline$b_{42, k}^{2}$ & $0.510^{-} 4$ & $1.510^{-} 4$ & $210^{-} 4$ \\
\hline
\end{tabular}

\section{REFERENCES}

[1] T. Varshney and S. Sheel, A Morlet wavelet neural network-based online identification and control of coupled MIMO systems, vol. 6, no. 3-4, pp. 246-260, 2012.

[2] J. Roll, A. Nazin and L. Ljung, Nonlinear system identification via direct weight optimization, Automatica, vol. 41, no. 3, pp. 475-490, 2005.

[3] I. K. Ibraheem and W. R. Abdul-Adheem, On the Improved Nonlinear Tracking Differentiator based Nonlinear PID Controller Design, International Journal of Advanced Computer Science and Applications, vol. 1, no. 7, pp. 234-241, 2016.

[4] H. K. Sahoo, P. K. Dash and N. P. Rath, NARX model based nonlinear dynamic system identification using low complexity neural networks and robust $H \infty$ filter, Applied Soft Computing, vol. 13, no. 7, pp. 3324-3334, 2013.

[5] L. Ljung, System identification: theory for the user, Upper Saddle River, New Jersey: Prentice Hall, 1999.

[6] J. Roll, A. Nazin and L. Ljung, Neural networks for nonlinear dynamic system modelling and identification, International journal of control, vol. 56, no. 2, pp. 319-346, 1992.

[7] T. Soderstrom and P. Stoica, System identification, Prentice Hall, 1989.

[8] X. Y. Kong, C. Z. Han, H. G. Ma and R. X. Wei, Fully Decoupled RLS Adaptive Identification Algorithm Based on Volterra Series, Acta Simulata Systematica Sinica, vol. 4, pp. 58-63, 2004.

[9] S. Boyd and L. Chua, Fading memory and the problem of approximating nonlinear operators with Volterra series, IEEE Transactions on circuits and systems, vol. 32, no. 11, pp. 1150-1161, 1985.
[10] S. L. Chang and T. Ogunfunmi, LMS/LMF and RLS Volterra system identification based on nonlinear Wiener model, In Circuits and Systems. ISCAS'98. Proceedings of the 1998 IEEE International Symposium, vol. 5, pp. 206-209, 1998.

[11] E. W. Bai and K. S. Chan, Identification of an additive nonlinear system and its applications in generalized Hammerstein models, Automatica, vol. 44, no. 2, pp. 430-436, 2008.

[12] S. Ai, S. Zheng, B. Mo and M. Yu, The hardware-in-the-loop simulation system of the diesel generator set based on the NARMAX model, Proceedings of the 2nd International Conference on Computer Science and Electronics Engineering, pp. 1080-1083, 2013.

[13] S. Rannen, C. Ghorbel and N. Benhadj Braiek, NARMAX structure and identification of coupled mass-spring-damper system, 3rd International Conference on Automation, Control, Engineering and Computer Science, pp. 475-480, 2016.

[14] S. Chen and S. A. Billings, Representations of non-linear systems: the NARMAX model, International Journal of Control, vol. 49, no. 3, pp. 1013-1032, 1989.

[15] R. Mtar, M. Belhaouane, M. Ayadi, H. Belkhiria and N. Benhadj Braiek, An LMI criterion for the global stability analysis of nonlinear polynomial systems, Nonlinear Dynamics and Systems Theory, vol. 9, no. 2, pp. 171183, 2009.

[16] N. Benhadj Braiek, H. Jribi and A. Becha, A Technique of a Stability Domain Determination for Nonlinear Discrete Polynomial Systems, The International Federation of Automatic Contol, IFAC, vol. 41, no. 2 pp. 8690-8694, 2008.

[17] A. Graham, Kronecker Products and Matrix Calculus: With Applications, John Wiley and Sons, 1982.

[18] N. Benhadj Braiek, Feedback stabilization and stability domain estimation of nonlinear systems, Journal of The Franklin Institute, vol. 332, no. 2, pp. 183-193, 1995.

[19] J. Sjoberg, Q. Zhang, L. Ljung, A. Benveniste, B. Delyon and P. Glorennec, Nonlinear black-box modeling in system identification: a unified overview, Automatica, vol. 31, no. 12, pp. 1691-1724, 1995.

[20] N. Benhadj Braiek, A Kronecker product approach of stability domain determination for nonlinear continuous systems, Systems Analysis Modelling Simulation, vol. 22, no. 1, pp. 11-16, 1996.

[21] S. Sundari and A. Nachiappan, Online identification using RLS algorithm and kaczmarzs projection algorithm for a bioreactor process, International Journal Of Engineering And Computer Science, vol. 3, pp. 7974-7978, 2014.

[22] C. Paleologu, J. Benesty and S. Ciochina, A robust variable forgetting factor recursive least-squares algorithm for system identification, IEEE Signal Processing Letters, vol. 15, pp. 597-600, 2008.

[23] A. Bemporad, M. Dua and E. N. Pistikopoulos, The explicit linear quadratic regulator for constrained systems, Systems Analysis Modelling Simulation, vol. 38, no. 1, pp. 3-20, 2002.

[24] W. R. Abdul-Adheem and I. K. Ibraheem, From PID to Nonlinear State Error Feedback Controller, International Journal of Advanced Computer Science and Applications, vol. 1, no. 8, pp. 312-322, 2017.

[25] T. H. Fay, FCoupled spring equations, International Journal of Mathematical Education in Science and Technology, vol. 34, pp. 65-79, 2003. 